\title{
What is the Short-Term Effect of a Dose of Antibiotics on Gut Microbiota in Thyroid Cancer Patients After Surgery? An Observational Clinical Study
}

\author{
Zengwu Yao ${ }^{1 \#}$, Yanbing Zhou ${ }^{2 \#}$, Lixin Jiang ${ }^{1 *}$, Yifei Zhang ${ }^{1}$, Jinchen $\mathrm{Hu}^{1}$, Zhiliang Wei ${ }^{2}$, Teng Sun ${ }^{2}, \mathrm{Yi} \mathrm{Li}^{2}$, \\ Xiaojie Wang ${ }^{1}$, Dawei Zhao ${ }^{1}$, Huiyuan Zhai ${ }^{1}$, Dong Wang ${ }^{1}$, Zhenbin Zhang ${ }^{1}$, Miaomiao Li $^{1}$ and Menglai \\ Zhang $^{1}$
}

${ }^{1}$ Gastrointestinal Surgery Ward I and Thyroid Surgery Ward, Yantai Yuhuangding Hospital, The Affiliated Hospital of Qingdao University, China

${ }^{2}$ Department of General Surgery, The Affiliated Hospital of Qingdao University, Qingdao, China

${ }^{\#}$ These authors contributed equally to this study.

\begin{abstract}
Background/Aim: International guidelines do not recommend routine systemic antibiotic prophylaxis in thyroidectomy patients as post-operative wound infections are rare, though many surgeons prefer to give preoperative antibiotics. However, the molecular-based short-term consequences of one dose of antibiotics on the human intestinal microbiota are rarely explored.

Materials and methods: We studied the short-term effects of preoperative antibiotics on the intestinal microbiota of three patients treated with a dose of cephazolin preoperatively and a non-treated patient who all underwent thyroidectomy due to thyroid cancer. The bacterial compositions in samples collected over a period of one month were monitored by analyzing the 16S rRNA gene using 454-based pyrosequencing.

Results: The microbial communities of four patients fluctuated over time, while the inter-individual difference showed the highest variability between all the samples. Taken as a whole, dramatic shifts in diversity and composition of gut microbiota after surgery compared to the preoperative states were not observed in the four patients who underwent thyroidectomy. However, at the genus level, the ratio of the two dominant genera Prevotella and Bacteroides showed significant change; Prevotella decreased while the ratio of Bacteroides increased greatly in most samples after surgery compared to the preoperative state in the study group and this was not observed in the patients who did not receive antibiotic treatment. Although antibiotic treatment, which is referred to as a major factor that disturbs the ecological balance between the human host and gut microbiota, can lead to the fluctuation of commensal gut microbiota, other factors such as host genetics, surgery, diet and environment, which may be confounded, may also shape the gut microbiota and should not be ignored.
\end{abstract}

\section{Keywords}

Antibiotics, Gut microbiota, Thyroid cancer, Short-term effect after surgery

It has been estimated that the microbes in our bodies collectively make up 100 trillion cells [1,2], ten times the number of total human cells and it is suggested that they encode 100 times more unique genes than our own genome encodes $[3,4]$. The human intestinal microbiota is essential to the health of the host and plays a role in nutrition, development, metabolism, pathogen resistance and regulation of immune responses, indeed, it is sometimes referred to as our "forgotten organ" $[5,6]$. Disruptions to the normal balance between the gut microbiota and the host have been associated with obesity, malnutrition, inflammatory bowel disease (IBD), neurological disorders and cancer [7].
*Corresponding author: Lixin Jiang, Gastrointestinal Surgery Ward I and Thyroid Surgery Ward, Yantai Yuhuangding Hospital, The Affiliated Hospital of Qingdao University, No.20 Yuhuangding East Road, Zhifu District, Yantai 264000, China, Tel: +8618105356668, Fax: +86-0535-6691999-83731

Accepted: June 22, 2019

Published online: June 24, 2019

Citation: Yao Z, Zhou Y, Jiang L, et al. (2019) What is the ShortTerm Effect of a Dose of Antibiotics on Gut Microbiota in Thyroid Cancer Patients After Surgery? An Observational Clinical Study. J Gastrointest Hepat Surg 2(1):18-29 
Citation: Yao Z, Zhou Y, Jiang L, et al. (2019) What is the Short-Term Effect of a Dose of Antibiotics on Gut Microbiota in Thyroid Cancer Patients After Surgery? An Observational Clinical Study. J Gastrointest Hepat Surg 2(1):18-29

Table 1: Features of participants and samples.

\begin{tabular}{|c|c|c|c|c|c|c|c|c|c|c|}
\hline \multicolumn{11}{|c|}{ Features of Participants and Samples } \\
\hline \multirow[t]{3}{*}{ Patient } & \multirow[t]{3}{*}{ Sex } & \multirow[t]{3}{*}{ Age } & \multirow[t]{3}{*}{ Country } & \multirow[t]{3}{*}{ Profession } & \multicolumn{6}{|c|}{ Samples Analyzed } \\
\hline & & & & & \multicolumn{3}{|c|}{ Preoperative } & \multicolumn{3}{|c|}{ Postoperative } \\
\hline & & & & & -1 & $1^{\text {st }}$ & $2^{\text {nd }}$ & 7 & 14 & 30 \\
\hline A & female & 31 & china & worker & 1 & 1 & 1 & 1 & 1 & 1 \\
\hline B & female & 34 & china & teacher & 1 & 1 & 1 & 1 & 1 & 1 \\
\hline C & female & 35 & china & doctor & 1 & 1 & 1 & 1 & 1 & 1 \\
\hline D & male & 40 & china & entrepreneur & 1 & 1 & 1 & 1 & 1 & 1 \\
\hline
\end{tabular}

Several factors such as host genetics, environment, diet and antibiotics can shape the human gut microbiota [7]. Antibiotics, especially broad-spectrum ones can affect not only the target pathogen, but also commensal inhabitants of the human host. Sometimes an imbalance in the commensal gut microbiota, due to antibiotic administration, can result in intestinal problems, such as antibiotic-associated diarrhoea (AAD) caused by Clostridium difficile and the expansion of antibiotic resistant strains [8-11].

To what extent disturbances occur depends on the spectrum of the antibiotics, the dose, the route of administration, pharmacokinetic and pharmacodynamic properties, and in vivo inactivation of antibiotics $[12,13]$. The direct analysis of genes encoding $16 \mathrm{~S}$ rRNA from gut microbiota has revealed that approximately $60 \sim 80 \%$ of the bacterial community in the intestine has not yet been cultured [14]. Based on this, recent studies indicated that a course of 7 days or 14 days antibiotic administration including clindamycin, ciprofloxacin, clarithromycin, metronidazole or amoxicillin could result in a longterm effect on the microbiota and could permanently prevent several of the taxa from returning to the pretreatment state within several months [15-19]. An important point to note is: studies on short-term impacts of cephalosporin on the gut microbiota mostly depend on culture-based approaches $[4,20,21]$, not molecular approaches.

International guidelines do not routinely recommend systemic antibiotic prophylaxis in clean surgical procedures such as thyroidectomy, since post-operative wound infections are rare [22]. Having said this, fulminant streptococcal wound infection after cervical operation did occasionally occur and carried a high mortality [23]. A multicentric randomized double-blind trial on patients who had undergone thyroidectomy indicated that the rate of surgical wound infection in patients who had undergone prophylactic antibiotic treatment was not statistically significant compared with those who had not [24]. An internet-based survey of all members of the International Association of Endocrine Surgeons concerning antibiotic prophylactics in thyroidectomy, parathyroidectomy, or neck dissections, has shown that the use of preoperative antibiotics varied widely: $62 \%$ of surgeons "almost never" used and $26.2 \%$ surgeons "almost always", and the prescribing behavior appeared dogmatic especially in Asia [25].

As previous reports have focused on the change of gut microbiota in gastrointestinal surgery $[20,26,27]$, little is known regarding the response of the microbiome of antibiotic pro- phylaxis in patients who have undergone non-gastrointestinal surgery. In the present study, we studied short-term effects of preoperative antibiotics on the intestinal microbiota of three patients who were treated with a dose of cephazolin and one patient who was not treated over a one-month time period. By using 16S rRNA gene pyrosequencing, we aimed to determine whether antibiotic treatment resulted in shortterm shifts in the microbial community structure in the samples.

\section{Material and Methods}

\section{Patients and treatment}

Four patients were selected for a thyroidectomy operation from Gastrointestinal Surgery Ward I and thyroid surgery Ward, Yantai Yuhuangding Hospital, the Affiliated Hospital of Qingdao University, China. None of the recruited patients had diabetes, infectious diseases, special diets, past allergic reactions to Cephalosporin antibiotics, were not pregnant or nursing. None of them had taken any antibiotics within the previous six months. Time series stool samples were collected at the following specific points: The day before surgery, the first and second defecating time, week 1 , week 2 and 1 month after surgery (Table 1 ). Patients $A, B$ and $C$ were treated with prophylaxis antibiotic (intravenous injection of 1 gram cefazolin30 minutes before anaesthesia). No prophylaxis antibiotic treatment was given to Patient $D$. This study was approved by the Ethics Committee of Yantai Yuhuangding Hospital, the Affiliated Hospital of Qingdao University, China; documented informed consent was obtained from all study participants. Participants were requested to report any symptoms co-occurring after operation, including mild gastrointestinal symptoms.

\section{Sample collection}

None of the patients were taking medication at the time of sample collection. Patients were asked to provide a frozen stool sample. Fresh stool samples were obtained at home and immediately frozen by storing them at $-20^{\circ} \mathrm{C}$ in their home freezer. Frozen samples were delivered to the Hospital using insulating polystyrene foam containers, and then were stored at $-80^{\circ} \mathrm{C}$ until analysis.

\section{DNA extraction}

A frozen aliquot ( $200 \mathrm{mg}$ ) of each stool sample was suspended in $250 \mu \mathrm{l}$ of guanidine thiocyanate, $0.1 \mathrm{M}$ Tris 
Citation: Yao Z, Zhou Y, Jiang L, et al. (2019) What is the Short-Term Effect of a Dose of Antibiotics on Gut Microbiota in Thyroid Cancer Patients After Surgery? An Observational Clinical Study. J Gastrointest Hepat Surg 2(1):18-29

(pH 7.5) and $40 \mu$ l of $10 \% \mathrm{~N}$-lauroyl sarcosine. Then, DNA extraction was conducted as previously described [28].

\section{Pyrosequencing}

PCR amplification of the V3 V5 region of bacterial $16 \mathrm{~S}$ rDNA was performed using universal primers (357F 5'-CCTACGGGNGGCWGCAG-3'; 926R 5'- CCGTCAATTCMTTTRAGT-3') incorporating the FLX Titanium adapters (A adapter sequence: 5' CCATCTCATCCCTGCGTGTCTCCGACTCAG 3'; B adapter sequence: $5^{\prime}$ CCTATCCCCTGTGTGCCTTGGCAGTCTCAG $3^{\prime}$ ) and a $10 \mathrm{bp}$ barcode sequence. The reagents and conditions of PCR are listed in Table 2. Following the PCR, amplicons were purified using the AMPure beads. Emulsion PCR and sequencing were performed according to the manufacturer's recommendations [2].

\section{Data analysis}

Part 1: Processing and quality filtering of raw sequence data: Sequences were processed using Mothur (version 1.27). Sequences were removed from the analysis if they were < $200 \mathrm{nt}$ or $>600 \mathrm{nt}$, had a read quality score $<30$, contained ambiguous characters, had more than $1 \mathrm{bp}$ barcode mismatch or contained more than 2 mismatches to the forward primer sequence $(357 \mathrm{~F})$. The high quality sequences were assigned to samples based on barcode matches.

Part 2: Taxonomic assignment of $16 \mathrm{~S}$ rDNA reads. 16S sequences were aligned with the SILVA database by using Mothur (version 1.27) http://schloss.micro.umass.edu/ [29]. A locally run version of RDP classifier (trainset9_032012) software was used to classify the sequences according to the methods proposed by Liu, et al. [30], operational taxonomic units (OTUs) were clustered with cut-off of $97 \%$. Diversity (Shannon and Simpson Index), richness (Chao1 and ACE) and

Table 2: The reagents and conditions of PCR.

\begin{tabular}{|c|c|}
\hline Reagents & Single dose ( $\mu \mathrm{l})$ \\
\hline Molecular Biology Grade Water & 35.2 \\
\hline $10 \times$ PFX buffer & 5 \\
\hline $\mathrm{MgSO}_{4}$ & 2 \\
\hline dNTPs (10 mM each) & 2 \\
\hline Fusion Primer A (10 $\mu \mathrm{M})$ & 2 \\
\hline Fusion Primer B $(10 \mu \mathrm{M})$ & 2 \\
\hline PFX polymerase & 0.8 \\
\hline
\end{tabular}

PCR conditions: $94{ }^{\circ} \mathrm{C}, 3 \mathrm{~min} ;\left(94{ }^{\circ} \mathrm{C}, 30 \mathrm{~s} ; 50{ }^{\circ} \mathrm{C}, 30 \mathrm{~s} ; 70{ }^{\circ} \mathrm{C}, 45 \mathrm{~s}\right)$ *30cycle; $70^{\circ} \mathrm{C}, 7 \mathrm{~min} ; 10^{\circ} \mathrm{C}$.

Table 3: Number of reads, OTUs and genera per patient $(A \sim D)$.

\begin{tabular}{|c|c|c|c|c|}
\hline Individual & Total no of reads & Analyzable reads & No. of OTUs & $\begin{array}{l}\text { No. of OTUs taxonomically classified } \\
\text { to a genus }\end{array}$ \\
\hline A1 & 5298 & 2398 & 170 & 111 \\
\hline$A 2$ & 8599 & 3391 & 187 & 123 \\
\hline A3 & 5293 & 1770 & 158 & 105 \\
\hline A4 & 10365 & 3901 & 193 & 114 \\
\hline A5 & 6237 & 2376 & 180 & 115 \\
\hline A6 & 11647 & 4878 & 236 & 146 \\
\hline B1 & 7497 & 3320 & 110 & 91 \\
\hline B2 & 7841 & 3406 & 138 & 109 \\
\hline B3 & 7586 & 3480 & 101 & 88 \\
\hline B4 & 10830 & 4361 & 146 & 116 \\
\hline B5 & 5635 & 2211 & 108 & 87 \\
\hline B6 & 10370 & 4774 & 141 & 111 \\
\hline $\mathrm{C} 1$ & 9326 & 4396 & 111 & 91 \\
\hline $\mathrm{C} 2$ & 7897 & 3338 & 120 & 105 \\
\hline C3 & 14247 & 6439 & 137 & 116 \\
\hline C4 & 12882 & 5751 & 128 & 102 \\
\hline C5 & 13169 & 5807 & 130 & 108 \\
\hline C6 & 9644 & 4221 & 123 & 103 \\
\hline D1 & 8192 & 3608 & 110 & 93 \\
\hline D2 & 9878 & 4235 & 146 & 114 \\
\hline D3 & 6894 & 3086 & 84 & 71 \\
\hline D4 & 7085 & 3139 & 103 & 89 \\
\hline D5 & 10349 & 4611 & 122 & 102 \\
\hline D6 & 5685 & 2369 & 107 & 88 \\
\hline
\end{tabular}


Citation: Yao Z, Zhou Y, Jiang L, et al. (2019) What is the Short-Term Effect of a Dose of Antibiotics on Gut Microbiota in Thyroid Cancer Patients After Surgery? An Observational Clinical Study. J Gastrointest Hepat Surg 2(1):18-29

Rarefaction curve analysis were also performed with OTUs with the above default identity threshold by Mothur (version 1.27). The heat map was constructed and analyzed using $R$ gplots package (version 2.15.2) based on the genera profiles of seven groups [31]. Unweighted and weighted UniFrac distance metrics analysis and principal component analysis (PCA) was conducted according to the matrix of distance using several custom $\mathrm{R}$ scripts.

\section{Results}

We monitored bacterial communities in the distal gut of three patients $(A, B$, and $C$ ) who were treated with antibiotic prophylaxis and a patient (D) who was not treated, by collecting stool samples ( 6 per patient) over a month period (Table 1).

\section{Sequencing depth and community diversity}

By $16 \mathrm{~S}$ rDNA pyrosequencing, we recovered a total of $\sim 91,000$ reads (1770 6439 per sample) from the 24 fecal samples. The pyrosequencing reads were clustered into 631 operational taxonomic units (OTUs; 101 236 per sample) (Table 3). For analysis of the pyrosequencing data, the proportions of reads classified into each taxonomic rank were first determined in four patients (Table 4). About 43\% 62\% of the reads in each patient were affiliated with the phylum
Firmicutes, 32\% 54\% with Bacteroidetes, 0.5\% 2.4\% with Proteobacteria, 0 1.8\% with Actinobacteria and fewer than 2.2\% with other phyla (Table 5 and Figure 1).

The Pie charts show the phyla found in stool samples. Numbers 1 6 represent different collecting time points: the day before surgery, the first and second defecating times, week 1 , week 2 and 1 month after surgery from three antibiotic prophylaxis treated patients $(A, B, C)$ and one patient who was not treated (D), respectively.

In the antibiotic prophylaxis group, after 1 week of intake, we observed an apparent decrease of Phylum Bacteroidetes and an increase of Phylum Firmicutes compared to the preoperation samples, which was not observed in the control group. By 1 month after surgery, they returned to their initial state more slowly in patient $C$ than in the other patients (Figure 1).

More than $50 \%$ OTUs could be classified to the genus level in all patients except patient A (Table 4). A heat map illustrated all genera (Figure 2), which indicated that the relative abundance of every genus in all samples tended to be more abundant between inter-individual than intraindividual, while the dominant genera were similar in all four patients. The top ten dominant genera in each patient

Table 4: The proportion of reads classified into different levels in each simple.

\begin{tabular}{|c|c|c|c|c|c|c|c|}
\hline & Domain & phylum & Class & Order & Family & Genus & Species \\
\hline A1 & $100.00 \%$ & $97.12 \%$ & $92.74 \%$ & $92.04 \%$ & $76.52 \%$ & $54.38 \%$ & $51.75 \%$ \\
\hline $\mathrm{A} 2$ & $100.00 \%$ & $97.08 \%$ & $87.44 \%$ & $86.97 \%$ & $65.73 \%$ & $43.23 \%$ & $34.30 \%$ \\
\hline A3 & $100.00 \%$ & $97.68 \%$ & $91.64 \%$ & $90.62 \%$ & $74.97 \%$ & $60.06 \%$ & $53.28 \%$ \\
\hline A4 & $100.00 \%$ & $95.90 \%$ & $80.36 \%$ & $79.59 \%$ & $52.96 \%$ & $38.89 \%$ & $28.61 \%$ \\
\hline A5 & $100.00 \%$ & $94.57 \%$ & $82.28 \%$ & $79.50 \%$ & $60.48 \%$ & $40.19 \%$ & $29.17 \%$ \\
\hline A6 & $100.00 \%$ & $93.79 \%$ & $86.20 \%$ & $84.26 \%$ & $70.87 \%$ & $55.47 \%$ & $50.43 \%$ \\
\hline B1 & $100.00 \%$ & $99.55 \%$ & $98.83 \%$ & $98.55 \%$ & $96.33 \%$ & $77.29 \%$ & $74.64 \%$ \\
\hline B2 & $100.00 \%$ & $99.24 \%$ & $98.65 \%$ & $98.30 \%$ & $95.65 \%$ & $69.47 \%$ & $66.53 \%$ \\
\hline B3 & $100.00 \%$ & $99.74 \%$ & $99.37 \%$ & $99.02 \%$ & $98.62 \%$ & $78.25 \%$ & $75.98 \%$ \\
\hline B4 & $100.00 \%$ & $96.56 \%$ & $94.98 \%$ & $94.54 \%$ & $91.36 \%$ & $69.30 \%$ & $62.30 \%$ \\
\hline B5 & $100.00 \%$ & $98.78 \%$ & $97.33 \%$ & $95.34 \%$ & $91.59 \%$ & $67.93 \%$ & $64.45 \%$ \\
\hline B6 & $100.00 \%$ & $98.81 \%$ & $98.18 \%$ & $96.75 \%$ & $95.58 \%$ & $70.61 \%$ & $68.12 \%$ \\
\hline $\mathrm{C} 1$ & $100.00 \%$ & $99.70 \%$ & $97.66 \%$ & $96.66 \%$ & $94.54 \%$ & $78.46 \%$ & $76.75 \%$ \\
\hline $\mathrm{C} 2$ & $100.00 \%$ & $99.67 \%$ & $98.95 \%$ & $98.05 \%$ & $95.96 \%$ & $66.18 \%$ & $60.90 \%$ \\
\hline C3 & $100.00 \%$ & $99.69 \%$ & $98.88 \%$ & $97.76 \%$ & $95.03 \%$ & $66.25 \%$ & $64.70 \%$ \\
\hline $\mathrm{C} 4$ & $100.00 \%$ & $99.57 \%$ & $98.38 \%$ & $97.24 \%$ & $93.08 \%$ & $70.02 \%$ & $68.67 \%$ \\
\hline C5 & $100.00 \%$ & $99.86 \%$ & $97.99 \%$ & $96.95 \%$ & $92.58 \%$ & $68.40 \%$ & $65.95 \%$ \\
\hline C6 & $100.00 \%$ & $99.79 \%$ & $97.70 \%$ & $97.35 \%$ & $94.12 \%$ & $74.08 \%$ & $71.97 \%$ \\
\hline D1 & $100.00 \%$ & $99.78 \%$ & $96.09 \%$ & $95.81 \%$ & $90.52 \%$ & $67.43 \%$ & $63.00 \%$ \\
\hline D2 & $100.00 \%$ & $99.69 \%$ & $98.77 \%$ & $98.02 \%$ & $88.93 \%$ & $58.91 \%$ & $56.95 \%$ \\
\hline D3 & $100.00 \%$ & $99.81 \%$ & $98.28 \%$ & $97.96 \%$ & $87.82 \%$ & $72.16 \%$ & $70.51 \%$ \\
\hline D4 & $100.00 \%$ & $99.78 \%$ & $99.39 \%$ & $99.11 \%$ & $90.60 \%$ & $73.81 \%$ & $72.09 \%$ \\
\hline D5 & $100.00 \%$ & $99.65 \%$ & $99.28 \%$ & $98.70 \%$ & $85.08 \%$ & $59.92 \%$ & $56.60 \%$ \\
\hline D6 & $100.00 \%$ & $99.70 \%$ & $97.09 \%$ & $96.41 \%$ & $87.08 \%$ & $69.78 \%$ & $67.24 \%$ \\
\hline
\end{tabular}


Citation: Yao Z, Zhou Y, Jiang L, et al. (2019) What is the Short-Term Effect of a Dose of Antibiotics on Gut Microbiota in Thyroid Cancer Patients After Surgery? An Observational Clinical Study. J Gastrointest Hepat Surg 2(1):18-29

Table 5: Individual relative abundance values (\%) for dominant phyla found in fecal samples over time.

\begin{tabular}{|c|c|c|c|c|c|c|c|c|c|c|c|c|}
\hline \multirow[b]{2}{*}{ Phylum } & \multicolumn{6}{|c|}{ Patient A } & \multicolumn{6}{|c|}{ Patient B } \\
\hline & $\mathrm{A} 1$ & $\mathrm{~A} 2$ & A3 & A4 & A5 & A6 & B1 & B2 & B3 & B4 & B5 & B6 \\
\hline Firmicutes & $54.4 \%$ & $63.0 \%$ & $63.2 \%$ & $68.9 \%$ & $68.5 \%$ & $56.0 \%$ & $37.1 \%$ & $42.2 \%$ & $31.2 \%$ & $55.8 \%$ & $44.8 \%$ & $44.0 \%$ \\
\hline Bacteroidetes & $41.9 \%$ & $31.7 \%$ & $33.1 \%$ & $22.4 \%$ & $24.5 \%$ & $36.4 \%$ & $61.1 \%$ & $54.8 \%$ & $66.3 \%$ & $36.8 \%$ & $51.6 \%$ & $53.6 \%$ \\
\hline Proteobacteria & $0.4 \%$ & $0.7 \%$ & $0.5 \%$ & $1.0 \%$ & $0.8 \%$ & $0.5 \%$ & $0.9 \%$ & $2.1 \%$ & $2 \%$ & $3.5 \%$ & $2.9 \%$ & $0.5 \%$ \\
\hline Actinobacteria & $0.2 \%$ & $0.7 \%$ & $0.7 \%$ & $1.8 \%$ & $0.5 \%$ & $0.8 \%$ & $0.3 \%$ & $0.1 \%$ & $0.1 \%$ & $0.4 \%$ & 0 & 0 \\
\hline \multirow[t]{2}{*}{ Other phyla } & $0.4 \%$ & $1.4 \%$ & $0.7 \%$ & $2.2 \%$ & $0.8 \%$ & $0.7 \%$ & $0.2 \%$ & $0.1 \%$ & $0.1 \%$ & $0.1 \%$ & $0.7 \%$ & $0.9 \%$ \\
\hline & \multicolumn{6}{|c|}{ Patient C } & \multicolumn{6}{|c|}{ Patient D } \\
\hline Phylum & $\mathrm{C} 1$ & $\mathrm{C} 2$ & C3 & $\mathrm{C} 4$ & C5 & $\mathrm{C} 6$ & D1 & D2 & D3 & D4 & D5 & D6 \\
\hline Firmicutes & $36.0 \%$ & $61.1 \%$ & $55.0 \%$ & $46.7 \%$ & $55.1 \%$ & $54.1 \%$ & $54.9 \%$ & $57.9 \%$ & $40.3 \%$ & $37.2 \%$ & $54.6 \%$ & $40.9 \%$ \\
\hline Bacteroidetes & $61.2 \%$ & $33.6 \%$ & $42.3 \%$ & $50.9 \%$ & $42.6 \%$ & $44.3 \%$ & $44.3 \%$ & $41.2 \%$ & $58.6 \%$ & $61.8 \%$ & $43.6 \%$ & $58.1 \%$ \\
\hline Proteobacteria & $2.6 \%$ & $4.7 \%$ & $2.2 \%$ & $1.9 \%$ & $2.0 \%$ & $1.1 \%$ & $0.1 \%$ & $0.1 \%$ & $0.7 \%$ & $0.7 \%$ & $1 \%$ & $0.5 \%$ \\
\hline Actinobacteria & 0 & $0.1 \%$ & $0.2 \%$ & $0.1 \%$ & $0.2 \%$ & $0.2 \%$ & $0.5 \%$ & $0.4 \%$ & $0.2 \%$ & $0.1 \%$ & $0.4 \%$ & $0.1 \%$ \\
\hline Other phyla & 0 & $0.1 \%$ & 0 & 0 & 0 & 0 & $0.1 \%$ & $0.3 \%$ & $0.2 \%$ & $0.1 \%$ & $0.3 \%$ & $0.2 \%$ \\
\hline
\end{tabular}

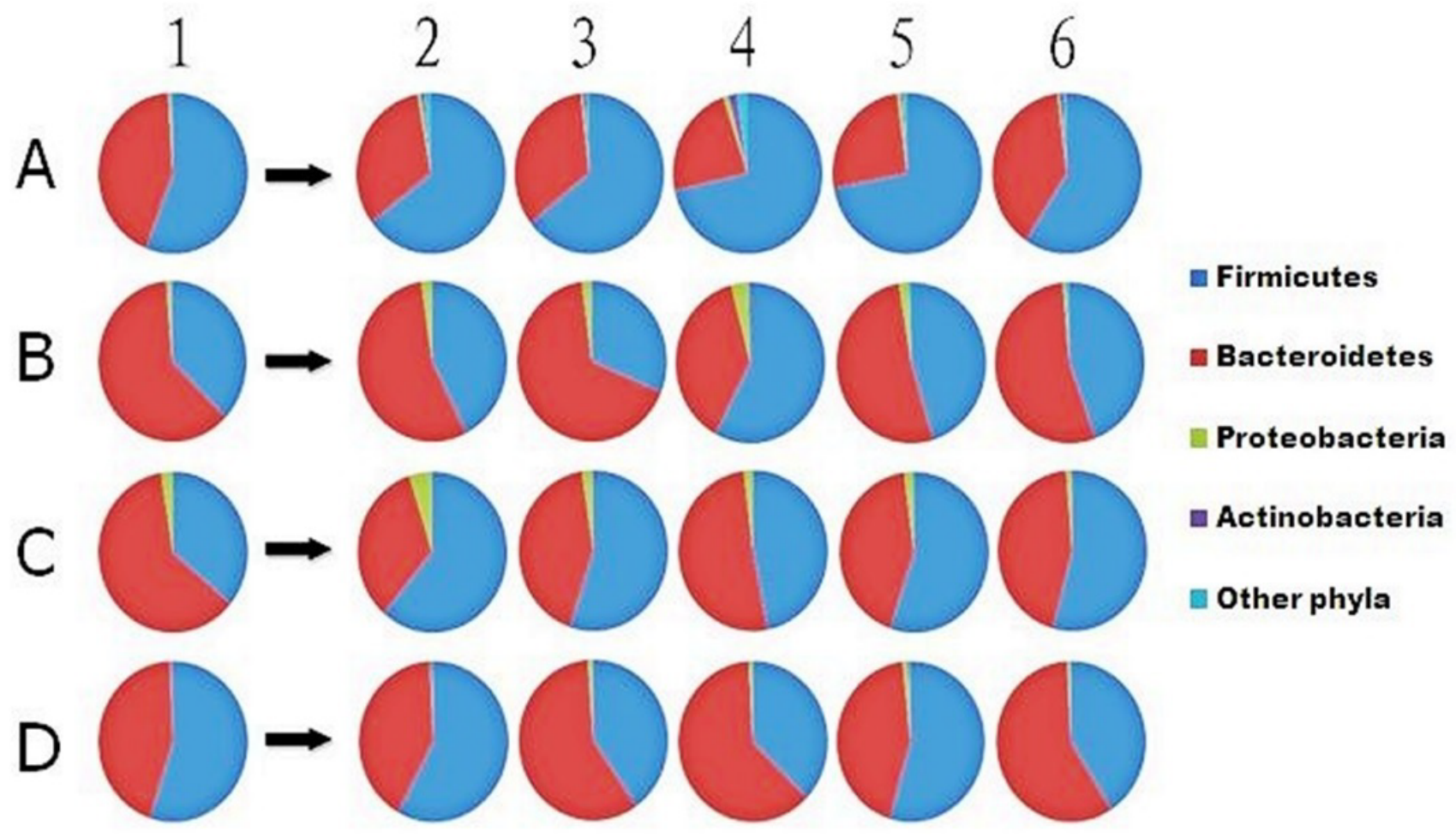

Figure 1: Phyla distribution in 24 stool samples.

was Prevotella, Bacteroides, unclassified Ruminococcaceae, Lachnospiracea_incertae_sedis, unclassified Lachnospiraceae, Dialister, Subdoligranulum, Alistipes, Coprococcus, Roseburia, Ruminococcus, Catenibacterium and Phascolarctobacterium (Figure 2 and Figure 3). However, these genera did not maintain uniform abundance after surgery.

The heat map shows the relative abundance per sample of 81 different genera in 9 phyla found in all stool samples. The color panel shows the percent relative abundance ( $0 \sim 56 \%$ ) of genera detected on different collecting time points in all the samples of patients (A D).
In addition to the overall trends in community change, the pyrosequencing data showed that specific members of the microbiota of each patient were impacted differently after surgery (Figure 3), presumably due to their unique microbiota compositions prior to treatment. In general, at the genus level, the negatively affected taxonomic group was Prevotella, and the positively affected group was Bacteroides in most samples of patients A, B and C (Figure 3). Each patient is separately discussed in the following section.

Figure 3 Each patient would be separately discussed in the following section. 
Citation: Yao Z, Zhou Y, Jiang L, et al. (2019) What is the Short-Term Effect of a Dose of Antibiotics on Gut Microbiota in Thyroid Cancer Patients After Surgery? An Observational Clinical Study. J Gastrointest Hepat Surg 2(1):18-29

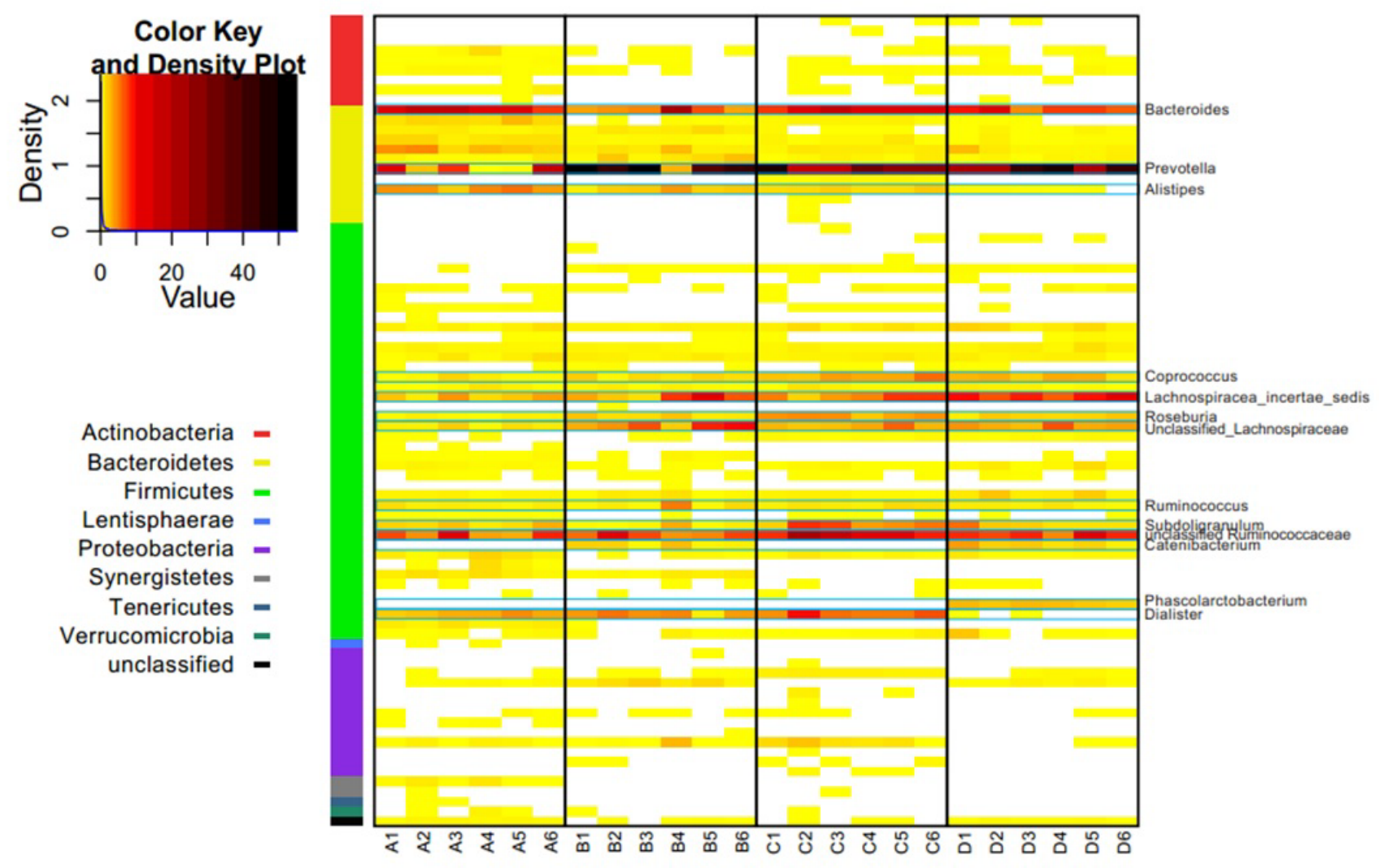

Figure 2: Genera distribution in 24 stool samples.

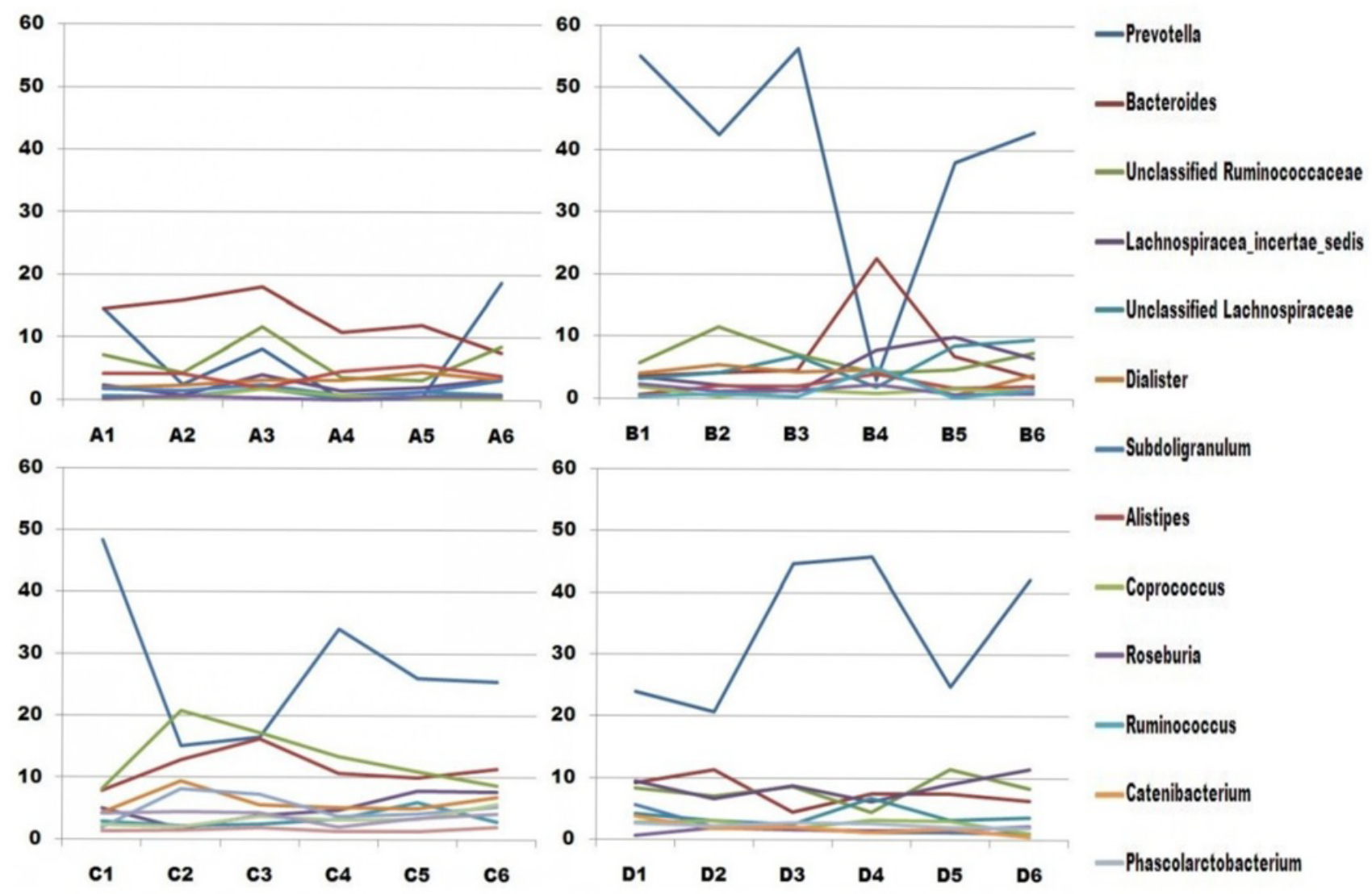

Figure 3: The variation of percentages of the top 10 genera in the samples of each patient over the collecting time. 
Citation: Yao Z, Zhou Y, Jiang L, et al. (2019) What is the Short-Term Effect of a Dose of Antibiotics on Gut Microbiota in Thyroid Cancer Patients After Surgery? An Observational Clinical Study. J Gastrointest Hepat Surg 2(1):18-29

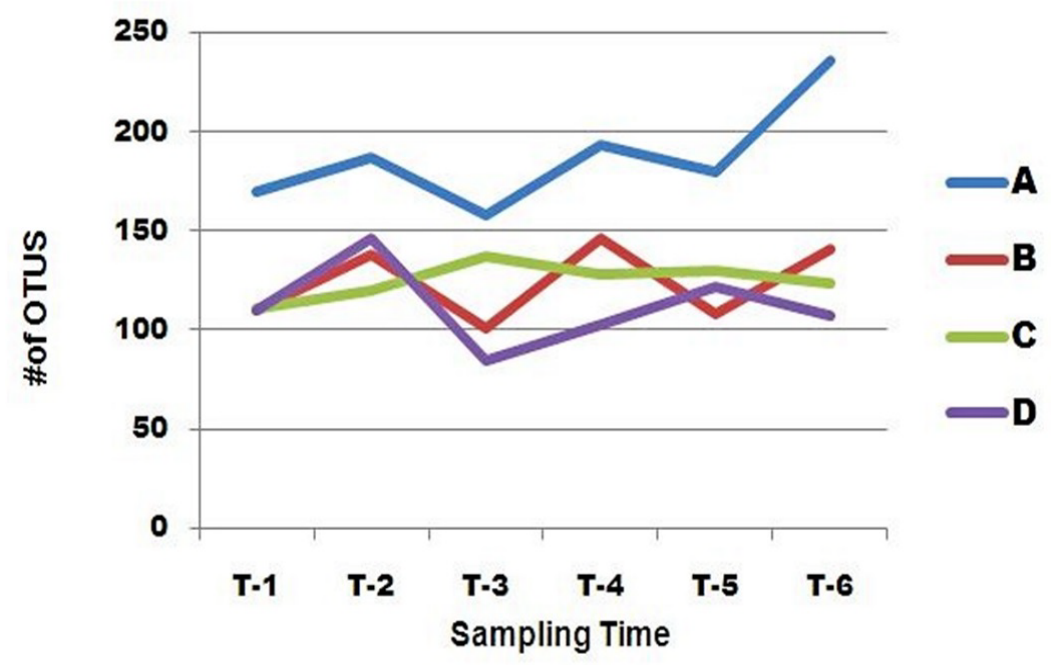

Figure 4: Curved line of fluctuation in the number of OTUs of all the samples of four patients over the collecting time.
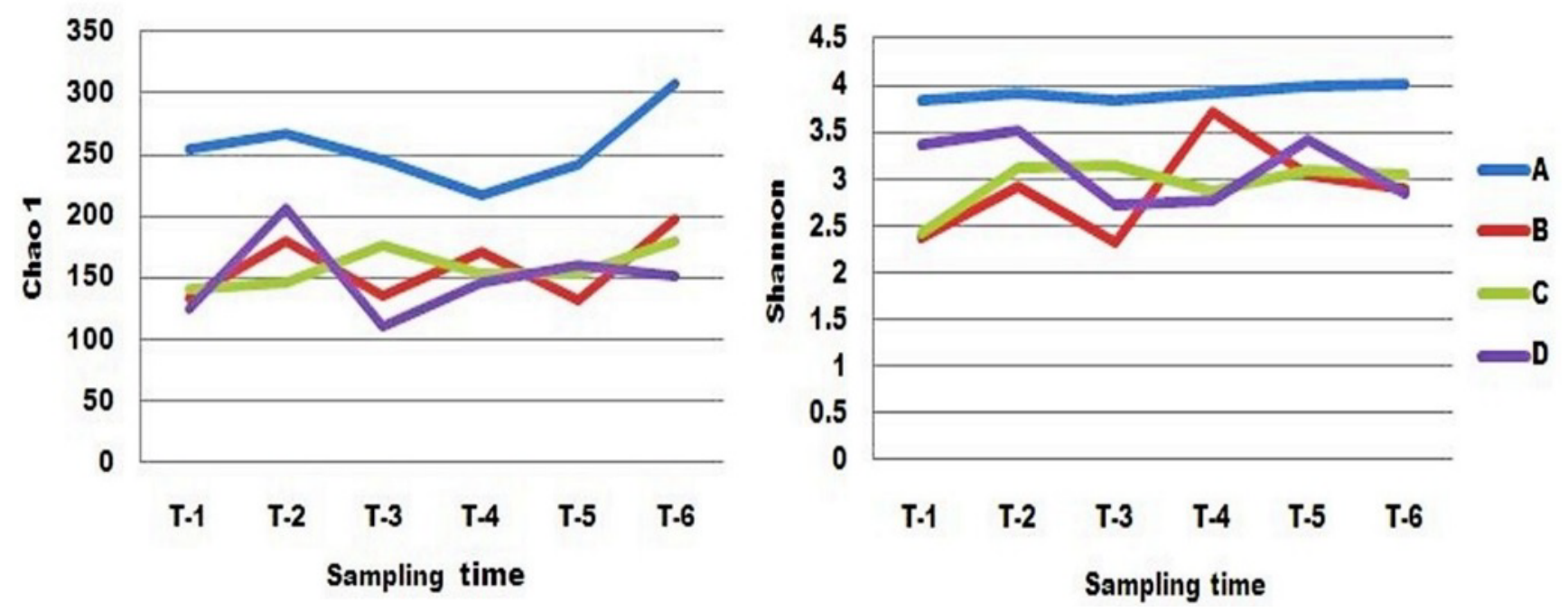

Figure 5: Curved line of change in Alpha diversity (Chao1 and Shannon index) of all the samples of four patients over the collecting time.

\section{Patient A}

The proportion of reads classified under the genus level in all samples of patient $A$ was the lowest, about $30 \% \sim 50 \%$ (Table 4). The proportion of top 10 genera mostly increased in the second defecating time after surgery. Prevotella decreased within two weeks after surgery, and recovered to its initial state at 1 month post surgery. The dominant genus, Bacteroides, increased slightly in the first and second postoperation samples, and then decreased later (from $18 \%$ to $8 \%)$.

\section{Patient B}

This sample was collected at week 1 post-operation, and the dominant genera, Prevotella and Bacteroides changed greatly. Prevotella decreased greatly (from 55\% to 3\%), and basically recovered at 1 month post-operation. Bacteroides increased (from $4 \%$ to $22 \%$ ), and recovered largely a week later. Other genera were relatively stable within the collecting period.

\section{Patient C}

A proportion of about $50 \%$ abundance, Prevotella, decreased at the first and second collection time after surgery, and then began to recover, but it did not recover to its initial state at 1 month post-operation. Bacteroides, unclassified Ruminococcaceae, Dialister and Subdoligranulum had a rising trend at the first or second defecating time after surgery, and returned to initial state at 1 month post-operation. Other genera were relatively stable.

\section{Patient D}

The dominant genus, Prevotella, increased greatly at the second defecating time and 1 week after surgery, recovered to its initial state at week 2 post-operation, and then increased. Unclassified Ruminococcaceae, Lachnospiracea_incertae sedis, and Bacteroides fluctuated more greatly in comparison 
Citation: Yao Z, Zhou Y, Jiang L, et al. (2019) What is the Short-Term Effect of a Dose of Antibiotics on Gut Microbiota in Thyroid Cancer Patients After Surgery? An Observational Clinical Study. J Gastrointest Hepat Surg 2(1):18-29

Table 6: Diversity index (chao1, ACE, Shannon, Simpson) in all the sample.

\begin{tabular}{|l|l|l|l|l|}
\hline Sample name & Chao1 & ACE & Shannon & Simpson \\
\hline A1 & 254.91 & 309.57 & 3.84 & 0.04 \\
\hline A2 & 267.64 & 323.10 & 3.92 & 0.04 \\
\hline A3 & 245.14 & 286.57 & 3.85 & 0.04 \\
\hline A4 & 217.41 & 227.22 & 3.91 & 0.04 \\
\hline A5 & 241.22 & 278.62 & 3.98 & 0.04 \\
\hline A6 & 307.72 & 302.47 & 4.02 & 0.05 \\
\hline B1 & 134.00 & 163.52 & 2.36 & 0.29 \\
\hline B2 & 179.35 & 170.77 & 2.92 & 0.18 \\
\hline B3 & 134.83 & 131.72 & 2.33 & 0.30 \\
\hline B4 & 170.47 & 174.19 & 3.71 & 0.05 \\
\hline B5 & 132.17 & 139.10 & 3.04 & 0.15 \\
\hline B6 & 196.65 & 219.46 & 2.90 & 0.18 \\
\hline C1 & 140.00 & 140.56 & 2.42 & 0.25 \\
\hline C2 & 145.14 & 173.95 & 3.11 & 0.09 \\
\hline C3 & 176.05 & 217.57 & 3.15 & 0.08 \\
\hline C4 & 152.17 & 155.94 & 2.87 & 0.15 \\
\hline C5 & 153.62 & 162.09 & 3.09 & 0.10 \\
\hline C6 & 180.27 & 179.58 & 3.05 & 0.10 \\
\hline D1 & 124.13 & 133.10 & 3.37 & 0.07 \\
\hline D2 & 206.71 & 262.43 & 3.52 & 0.06 \\
\hline D3 & 110.25 & 100.57 & 2.71 & 0.16 \\
\hline D4 & 159.80 & 140.99 & 2.76 & 0.16 \\
\hline D5 & 147.27 & 3.42 & 0.06 \\
\hline D6 & 186.07 & 2.84 & 0.14 \\
\hline
\end{tabular}

\section{A)}

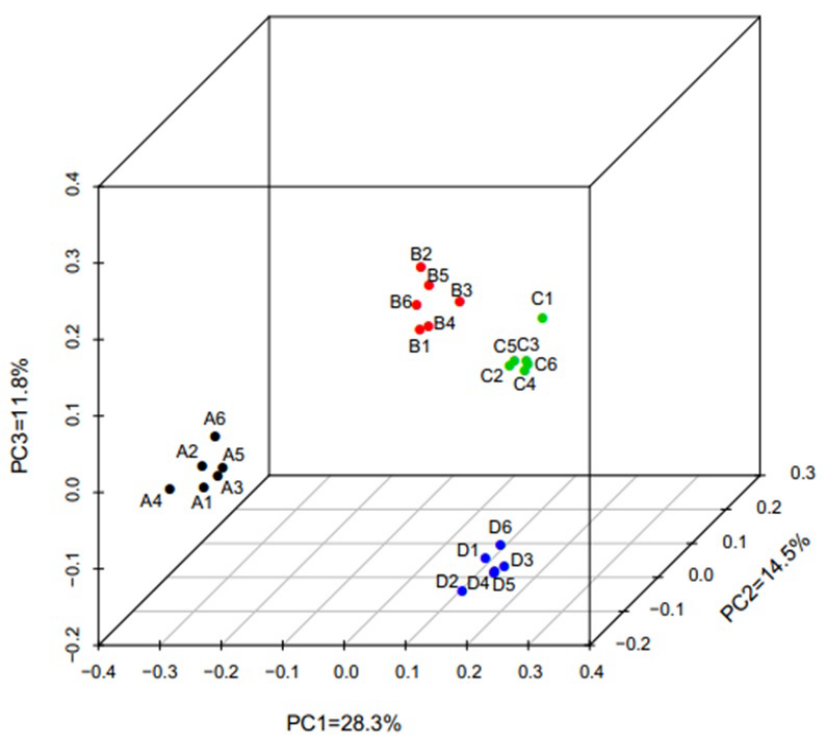

with other genera except Prevotella over a month period.

\section{Fluctuation in Diversity}

All the patients shared similar features across a one month period, as indicated by plots of OTUs richness (Figure 4). There was no obvious difference in fluctuation of the distal gut microbiota between patients A, B, C and patient D. Interestingly, the number of OTUs in distal gut microbiota across all the patients increased in the first sample collected after surgery compared to the sample collected before surgery, although the wave of increase was different, and then decreased in the second post-operation sample except in patient $C$. Patient $C$ was the most stable of all the patients. The lowest value of OTU richness occurred in the second sample collected after surgery in patients $A, B$ and $D$.

Alpha diversity of all the samples in the patients was estimated by Chao1, Shannon index over time (Table 6 and Figure 5). The Chao 1 richness estimators were used to estimate the total number of OTUs, so its variation was similar to the change of OTU richness. In contrast, patient $A$ was the most stable by Shannon index, and its value was the largest in all the patients over a one month period. Compared to the sample collected before surgery, the value of the first samples collected after surgery in patients $B, C$, and D increased, and then decreased. The displacement of the pre-operation value in patients B, C and D seemed larger within the first week after surgery than in future weeks.

\section{Fluctuation of community membership}

Similarities and differences between the membership of the distal gut microbial communities of the four patients are portrayed by principal coordinate analysis (PCOA) of unweighted UniFrac distances, a measure of community dissim-

Figure 6: Graphic model of principal coordinate analysis (PCOA) based on unweighted; A) and weighted UniFrac distances; B) in all the samples of 4 patients. 
ilarity based on OTU presence/absence (Figure 6A). Inter individual variation was the major source of variability between samples. Community membership of Patient B was similar to that of patient $C$, as their distance was smallest among all the samples of the four patients. However, the results were different in weighted UniFrac PCoA which was based on OTU relative abundance (Figure $6 B$ ). By using Weighted UniFrac PcoA, changes in the distance between the samples of each patient were more obvious, which indicated that the proportion of each OTU changed remarkably after surgery. Compared to the preoperative sample, the most similar one of post-operation samples in each patient was incomplete different. In particular, the sample collected at week 1 after surgery in patient B was obviously separated from the other samples, and its composition was similar to the samples of patient A (Figure 7).

We assessed the changing community composition during different time periods of the experiment by using Bray-Curtis inter sample distances (BC). BC is a common metric of community dissimilarity because it makes the reasonable assumption that the shared absence of a taxon is not evidence of community similarity. When the value of the $\mathrm{BC}$ is bigger, the communities are more different. Patient $D$ was the most stable over the colleting time of all the patients. In patients A, B and $D$, compared to the preoperative sample, the sample at week 1 after surgery was the most different, whereas in patient $C$ this was the most similar sample. In patient $B$, the sample collected at week 1 post-operation changed more greatly than any other samples of all the patients.

\section{Discussion}

We used molecular approaches to monitor and compare ecological disturbances of the human microbiome in thyroid carcinoma patients who were treated or untreated with antibiotic prophylaxis. We found that there was no dramatic shift of the gut microbiota in all the patients after surgery over a month's period. At the genus level the patient who received no antibiotic prophylaxis was relatively stable in membership of microbiota compared to the antibiotic prophylaxis group. The proportion of dominant genera in most samples after surgery, Prevotella and Bacteroides, changed in opposite directions compared with the preoperative samples in the antibiotic prophylaxis group, although the response of each patient was different. We also observed the resilience of the human gut microbiota after surgery. Inter individual difference was the largest source of variability between all the samples.

There were many reasons why no dramatic shifts in gut microbiota of all the patients were observed. The use of one dose of antibiotics might be the major reason and other factors included antimicrobial spectrums, pharmacological action and excretion of cefazolin, antibiotic-resistant genes in the Chinese population, change of diet, routine temporal variability and resilience of gut microbiota, human genetics, age, environment, surgery and so on.

The extent of disturbance of gut microbiota depends on the antimicrobial spectrums and doses of the antibiotic. Most of the reports were based on cultivation-dependent approaches. If we take ceftriaxone, a third generation cephalosporin as an example, it seemed that one dose of ceftriaxone had a more moderate effect on the gut microbiota compared to multi doses. A 10-day parenteral therapy with ceftriaxone in patients with community-acquired pneumonia caused a reduction in the number of Escherichia coli and an increase in the number of enterococci and yeasts. In the anaerobic microflora, lactobacilli, bifidobacteria, and clostridia were suppressed [32]. In contrast, the effect of a single dose of ceftriaxone just before anaesthesia in patients undergoing colorectal surgery was that in the intestinal flora, the number of enterobacteria was suppressed or eliminated. Only minor changes occurred in the Gram-positive aerobic flora and in the anaerobic microflora [20].

Only a handful of studies have used cultivation-indepen-

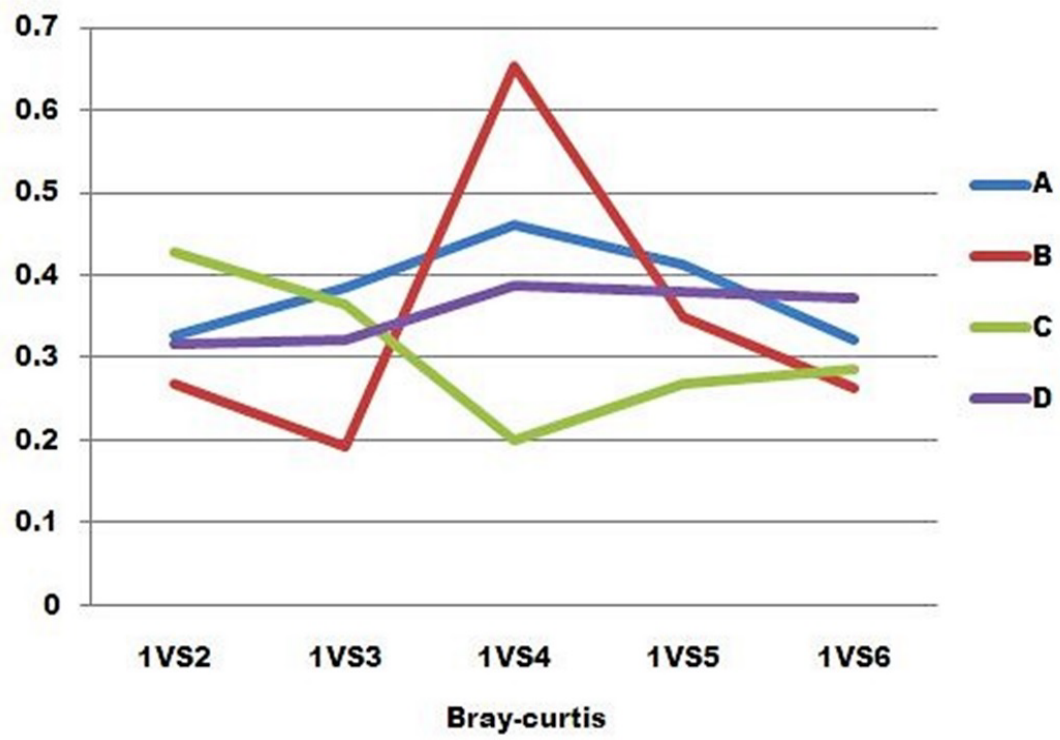

Figure 7: Bray-Curtis inter sample distances $(\mathrm{BC})$ between each sample after surgery and the preoperative sample in each patient. 
Citation: Yao Z, Zhou Y, Jiang L, et al. (2019) What is the Short-Term Effect of a Dose of Antibiotics on Gut Microbiota in Thyroid Cancer Patients After Surgery? An Observational Clinical Study. J Gastrointest Hepat Surg 2(1):18-29

dent surveys to examine the long-term consequences of multi doses of antibiotics, rather than one dose. These studies found that antibiotics obviously altered the composition of the gut microbiota, not only the targeted pathogen but also the commensal bacteria, and that the abundance of most taxa began to return to prior levels within several weeks and several taxa did not recover within six months [15-17]. In our study, we observed the one dose of cefazolin had no dramatic effect on gut microbiota, including the taxonomic richness, diversity, and evenness of the community within a month period.

Antibiotics shift the composition of the microbiota in different ways depending on their spectrum of activity. The spectrum of cefazolin covers the following pathogens: Staphylococcus aureus, Staphylococcus epidermidis, beta-hemolytic group A streptococci and other strains of streptococci; Diplococcus pneumoniae, E. coli, Proteus mirabilis, Klebsiella sp., Enterobacter aerogenes, and Haemophilus influenza [33]. In our study, we did not detect these pathogens because either the pathogen did not appear in the gut microbiota or the $16 \mathrm{~s}$ RNA pyrosequencing was limited in identifying every strain in the stool. At the same time, no gastrointestinal symptom was reported by any of the patients. The potentially pathogenic microbes associated with antibiotic-associated diarrhea such as Klebsiella oxytoca, Clostridium perfringens, Clostridium difficile and Staphylococcus aureus were not found in the current study [34].

Cefazolinis poorly absorbed from the gastrointestinal tract and more than $90 \%$ of its excretion is by the kidney, with a little through the bile [35]. The effect of cefazolinon the gut micriobiota was weak, as the drug did not go through the gastrointestinal tract directly.

Antibiotic resistance is a serious public health threat in the world [36] and, together with the dogmatic antibiotic prescribing behavior and heavy use of antibiotics in livestock have made China a special case [37]. For example, a study on the antibiotic use in 118 hospitals around China revealed that the proportion of prophylaxis antibiotics in clean surgery was about $96.9 \%$ with no indication on prescribing behavior [38]. Another survey in 2007 estimated that nearly half of the 210,000 tons of antibiotics produced in China end up in animal feed [39]. As a result, China had the world's most rapid growth rate of resistance [40], resulting in the government of China taking more serious measures to control the rampant antibiotic resistance.

Although the patients did not use antibiotics within at least six months before sample collection, we were not sure that their food intake did not include antibiotics. Elevated levels of antibiotic resistance genes might make the Chinese population more resistant to broad-spectrum antibiotics. That may be another reason why the patients had a moderate shift of gut microbiota responding to one dose of cefazolin. However, whether patients who would be exposed to antibiotics again would have a more obvious effect on their gut microbiota or not was unclear. We advised that doctors must strictly follow the guidelines of antibiotic use, and should not routinely use antibiotic prophylaxis in clean surgery, such as thyroidectomy, unless the patients had risk factors of wound infection. If not, there would be embarrassment at the emergence and prevalence of superbacteria which resulted from antibiotic overuse in the future.

Our results revealed that at the genus level, the dominant genera, Prevotella and Bacteroides, changed their proportions greatly in most postoperative samples. They correlated well with enterotypes of the human gut microbiome [41]. Bacteroides was associated with a long-term diet rich in animal protein, several amino acids and saturated fats, and Prevotella was associated with carbohydrates and simple sugars [42]. All the patients were fasting and were provided energy by venous transfusion on the operation day. Although all the patients had no particular diet, patients began to eat as usual on the first day after surgery, and the volume of food-intake largely recovered to preoperative levels on third or fourth day after surgery due to incision ache in the neck. So the change in volume of diet might partly explain why the dominant genus changed after surgery.

The resilience of the microbiota to antibiotic treatments varied between individuals [17], which was also observed in our current study. It was probably due to the known unique bacterial community compositions in different patients $[5,43,44]$. Species-rich communities are less susceptible to invasion because they use limiting resources more efficiently, with different species specialized to each potentially limiting resource [45]. The microbial communities were more similar within individuals than between individuals across the sampling period. It was consistent with the previous studies that the inter-individual variation was the major source of variation regardless of antibiotic [17].

The routine fluctuations in community composition might explain some of the variation after surgery and the shortterm stability of the distal gut community which varied within a certain range [17]. The range of routine fluctuations is unclear to us, and it may be different between individuals. A previous report has shown that most taxa changed in relative abundance from day to day, communities were no more different when compared at times separated by $2 \sim 5$ months, on average, than they were at times separated by more than about 1 week [17]. So no dramatic shifts of gut microbiota might be based on the routine fluctuations in community composition across a one month collecting period in our study. Other factors such as host genetics [46], environment and age $[44]$ and surgery $[4,27]$ might not be ignored. The fact that all the patients had no kinship and different professions might result in the inter-individual differences. All patients of ages 30 to 40 had a better ability to recover than the elderly [44].

In conclusion, a dose of cefazolin treatment resulted in moderate ecological disturbances in the gut microbiota in a short term period. Compared to conventional cultivation techniques, the molecular approaches that we used enabled a more detailed monitoring of ecological disturbances due to antibiotic treatment. In total, the observations in the present study underlined the moderate fluctuation of the human gut microbiota in patients who underwent thyroidectomy with 
antibiotic prophylaxis treatment versus those who did not. However, the number of patients was too small to obtain a useful statistical viewpoint. For future studies it would be valuable to analyze more samples before onset of treatment for each individual to establish a better baseline.

\section{Acknowledgements}

The bioinformatics analysis in this study were provided by Shenzhen Huada Gene Research Institute.

\section{References}

1. Peterson J, Garges S, Giovanni M, et al. (2009) The NIH human microbiome project. Genome Research 19: 2317-2323.

2. Jumpstart consortium human microbiome project data generation working group (2012) Evaluation of 16S rDNA-based community profiling for human microbiome research. PloS One 7: E39315.

3. Ley RE, Peterson DA, Gordon JI (2006) Ecological and evolutionary forces shaping microbial diversity in the human intestine. Cell 124: 837-848.

4. Bächer K, Schaeffer M, Lode H, et al. (1992) Multiple dose pharmacokinetics, safety, and effects on faecal microflora, of cefepime in healthy volunteers. J Antimicrob Chemother 30: 365-375.

5. Eckburg PB, Bik EM, Bernstein CN, et al. (2005) Diversity of the human intestinal microbial flora. Science 308: 1635-1638.

6. O'Hara AM, Shanahan F (2006) The gut flora as a forgotten organ. EMBO Reports 7: 688-693.

7. Lozupone CA, Stombaugh JI, Gordon JI, et al. (2012) Diversity, stability and resilience of the human gut microbiota. Nature 489 220-230.

8. Kelly CP, LaMont JT (2008) Clostridium difficile-more difficult than ever. N Engl J Med 359: 1932-1940.

9. De La Cochetiere MF, Durand T, Lalande V, et al. (2008) Effect of antibiotic therapy on human fecal microbiota and the relation to the development of Clostridium difficile. Microb Ecol 56: 395402.

10. Owens RC Jr, Donskey CJ, Gaynes RP, et al. (2008) Antimicrobialassociated risk factors for Clostridium difficile infection. Clin Infect Dis 46: S19-S31.

11. Beaugerie L, Petit JC (2004) Microbial-gut interactions in health and disease. Antibiotic-associated diarrhoea. Best Pract Res Clin Gastroenterol 18: 337-352.

12. Sullivan $\AA$, Edlund C, Nord CE (2001) Effect of antimicrobial agents on the ecological balance of human microflora. Lancet Infect Dis 1: 101-114.

13. Jernberg C, Lofmark S, Edlund C, et al. (2010) Long-term impacts of antibiotic exposure on the human intestinal microbiota. Microbiology 156: 3216-3223.

14. Suau A, Bonnet R, Sutren M, et al. (1999) Direct analysis of genes encoding 16S rRNA from complex communities reveals many novel molecular species within the human gut. Appl Environ Microbiol 65: 4799-4807.

15. Jakobsson HE, Jernberg C, Andersson AF, et al. (2010) Shortterm antibiotic treatment has differing long-term impacts on the human throat and gut microbiome. PloS One 5: E9836.
16. Dethlefsen L, Huse S, Sogin ML, et al. (2008) The pervasive effects of an antibiotic on the human gut microbiota, as revealed by deep 16S rRNA sequencing. PLoS Biol 6: E280.

17. Dethlefsen L, Relman DA (2011) Incomplete recovery and individualized responses of the human distal gut microbiota to repeated antibiotic perturbation. Proc Natl Acad Sci U S A 108: 4554-4561.

18. Jernberg C, Lofmark S, Edlund C, et al. (2007) Long-term ecological impacts of antibiotic administration on the human intestinal microbiota. ISME J 1: 56-66.

19. Janczyk P, Pieper R, Souffrant WB, et al. (2007) Parenteral long-acting amoxicillin reduces intestinal bacterial community diversity in piglets even 5 weeks after the administration. ISME J 1: 180-183.

20. Cavallaro V, Catania V, Bonaccorso R, et al. (1992) Effect of a broad-spectrum cephalosporin on the oral and intestinal microflora in patients undergoing colorectal surgery. J Chemother 4: 82-87.

21. Welling G, Meijer-Severs GJ, Helmus G, et al. (1991) The effect of ceftriaxone on the anaerobic bacterial flora and the bacterial enzymatic activity in the intestinal tract. Infection 19: 313-316.

22. De Lalla F (2002) Surgical prophylaxis in practice. J Hosp Infect 50: S9-S12.

23. Hardy R, Forsythe J (2007) Uncovering a rare but critical complication following thyroid surgery: An audit across the UK and Ireland. Thyroid 17: 63-65.

24. Avenia N, Sanguinetti A, Cirocchi R, et al. (2009) Antibiotic prophylaxis in thyroid surgery: A preliminary multicentric Italian experience. Annals of Surgical Innovation and Research 3: 10.

25. Moalem J, Ruan DT, Farkas RL, et al. (2010) Patterns of antibiotic prophylaxis use for thyroidectomy and parathyroidectomy: Results of an international survey of endocrine surgeons. J Am Coll Surg 210: 949-956.

26. Graessler J, Qin Y, Zhong H, et al. (2012) Metagenomic sequencing of the human gut microbiome before and after bariatric surgery in obese patients with type 2 diabetes: Correlation with inflammatory and metabolic parameters. Pharmacogenomics J 13: $514-522$.

27. Åberg C, Thore M (1991) Single versus triple dose antimicrobial prophylaxis in elective abdominal surgery and the impact on bacterial ecology. J Hosp Infect 18: 149-154.

28. Manichanh C, Rigottier-Gois L, Bonnaud E, et al. (2006) Reduced diversity of faecal microbiota in Crohn's disease revealed by a metagenomic approach. Gut 55: 205-211.

29. Schloss PD, Westcott SL, Ryabin T, et al. (2009) Introducing mothur: Open-source, platform-independent, communitysupported software for describing and comparing microbial communities. Appl Environ Microbiol 75: 7537-7541.

30. Liu Z, DeSantis TZ, Andersen GL, et al. (2008) Accurate taxonomy assignments from 16S rRNA sequences produced by highly parallel pyrosequencers. Nucleic Acids Res 36: E120-E120.

31. Luna A, Nicodemus KK (2007) snp plotter: An R-based SNP/ haplotype association and linkage disequilibrium plotting package. Bioinformatics 23: 774-776.

32. Vogel F, Ochs H, Wettich K, et al. (2001) Effect of step-down therapy of ceftriaxone plus loracarbef versus parenteral therapy of ceftriaxone on the intestinal microflora in patients with community-acquired pneumonia. Clin Microbiol Infect 7: 376379. 
Citation: Yao Z, Zhou Y, Jiang L, et al. (2019) What is the Short-Term Effect of a Dose of Antibiotics on Gut Microbiota in Thyroid Cancer Patients After Surgery? An Observational Clinical Study. J Gastrointest Hepat Surg 2(1):18-29

33. Weinstein A (1980) The cephalosporins: Activity and clinical use. Drugs 20: 137.

34. Bartlett JG (2002) Antibiotic-associated diarrhea. New England Journal of Medicine 346: 334-339.

35. Lanao J, Vicente M, Dominguez-Gil A (1988) Pharmacokinetics of cefazolin administered as a new drug delivery system in healthy volunteers. Biopharm Drug Dispos 9: 377-388.

36. Goossens $\mathrm{H}$, Ferech $\mathrm{M}$, Vander Stichele R, et al. (2005) Outpatient antibiotic use in Europe and association with resistance: A crossnational database study. The Lancet 365: 579-587.

37. Heddini A, Cars O, Qiang S, et al. (2009) Antibiotic resistance in China-a major future challenge. The Lancet 373: 30.

38. He SP, Li ZL, Yan Q (2008) Survey of the perioperative prophylactic use of antimicrobial agents in 118 hospitals in China. Zhonghua wai ke za zhi 46: 12-14.

39. Hvistendahl M (2012) Public health. China Takes Aim at Rampant Antibiotic Resistance. Science 336: 795-795.
40. Ruifang Z, Karen E, Vincent R, et al. (2006) Antibiotic resistance as a global threat: Evidence from China, Kuwait and the United States. Global Health 2: 6.

41. Arumugam M (2011) Enterotypes of the human gut microbiome. Nature 473: 174-180.

42. Wu GD, Chen J, Hoffmann C, et al. (2011) Linking long-term dietary patterns with gut microbial enterotypes. Science 334: 105-108.

43. Lozupone CA, Stombaugh JI, Gordon JI, et al. (2012) Diversity, stability and resilience of the human gut microbiota. Nature 489: 220-230.

44. Yatsunenko T, Rey FE, Manary MJ, et al. (2012) Human gut microbiome viewed across age and geography. Nature 486: 222227.

45. Levine JM, D'Antonio CM (1999) Elton revisited: A review of evidence linking diversity and invasibility. Oikos, 15-26.

46. Turnbaugh PJ, Hamady M, Yatsunenko T, et al. (2009) A core gut microbiome in obese and lean twins. Nature 457: 480-484. 